\title{
Signal regulatory protein $\alpha 1$ is involved in the inhibitory effect of glucocorticoid receptor on the proliferation of murine macrophage RAW264.7 cell and mouse peritoneal macrophage
}

\author{
Xiaohui Wang*, Yidong Li*, Xiaoyan Zhu, Yan Wang, Fei Diao and Jian Lu \\ Department of Pathophysiology, Second Military Medical University, 800 Xiangyin Road, Shanghai 200433, People's Republic of China \\ (Correspondence should be addressed to J Lu; Email: lujian326@ 163.com) \\ ${ }^{*}(\mathrm{X}$ Wang and $\mathrm{Y} \mathrm{Li}$ are co-first authors $)$
}

\begin{abstract}
Glucocorticoid (GC) effectively suppresses immune and inflammatory responses and inhibits the growth of several types of cells, but the role of GC and its receptor on macrophage proliferation is unclear. In our previous work, we found RAW$\mathrm{GR}(-)$ cells (murine macrophage RAW264.7 cells stably transfected with GR-siRNA expression vector by RNA interference) grew faster by about twofold. In this study, we further explored the role and mechanisms of GC/GR on the proliferation of macrophage. We found that the growth of RAW264.7 cells was inhibited by dexamethasone (Dex) in a concentration-dependent manner. The mRNA and protein levels of signal regulatory protein $\alpha 1$ (SIRPA) were induced by GC/GR in RAW264.7 cells and SIRPA expression was decreased remarkably in RAW-GR(-) cells. Overexpression of SIRPA negatively regulated the proliferation of RAW-GR $(-)$ cells, and inhibition of SIRPA expression by a small from RNA interference attenuated Dex-induced proliferation inhibition in RAW264.7 cells. The proliferation inhibition of GC/GR was also found in mouse peritoneal macrophage, which was associated with the increase in SIRPA induced by GC/GR as well. In addition, elevation of the expression of CDK2, cyclinD1, and cyclinB1, but not phosphorylated ERK1/2 and p38, was found in RAW-GR(-) cells. In conclusion, we provided the novel evidences that GC/GR inhibited the growth of RAW264.7 cells and mouse peritoneal macrophage, and the antiproliferative effect of GC/GR on these cells was at least in part a result from GC/GR-induced SIRPA expression. Up-regulation of CDK2, cyclinD1, and cyclinB1 was also related to the increased proliferation of RAW-GR(-) cells.
\end{abstract}

Journal of Molecular Endocrinology (2008) 41, 393-403

\section{Introduction}

It is well known that glucocorticoid (GC) plays a key role in regulating diverse physiological processes including immune and inflammatory responses, cell proliferation, differentiation, apoptosis, and development. These actions are mediated by glucocorticoid receptor (GR) that belongs to the nuclear receptor superfamily and is normally localized in the cytoplasm in an inactive state. On hormone binding, GR translocates to the nucleus where it binds to glucocorticoid response elements (GREs) on target genes and recruits coactivators or corepressors to regulate the expression of target genes (Heitzer et al. 2007).

GC is commonly used in the treatment of autoimmune and inflammatory diseases. It has been well documented that the inhibition of proliferation and induction of lymphocytic apopotosis are involved in the mechanisms of immunosuppressive effects of GCs (Distelhorst 2002, Frankfurt \& Rosen 2004). Macrophages also play a key role in the immune and inflammatory processes, mainly acting as antigen- processing/presenting cells and sources of inflammatory cytokines. Although GCs have been reported to inhibit macrophage killing activity and cytokine production in response to proinflammatory stimuli (Brummer et al. 2005), the effect of GCs on macrophage proliferation is controversial. Pagniello et al. (2002) found that dexamethasone (Dex) inhibited proliferation of the rainbow trout monocyte/macrophage cell line, RTS11. However Lloberas et al. (1998) reported that Dex itself had no effect on the proliferation of differentiated or undifferentiated bone marrow-derived macrophages and isolated peritoneal macrophages. In our previous study, we found that inhibition of GR expression in murine macrophage cell line RAW267.4 by RNA interference (RNAi) significantly promoted cell proliferation (Zhu et al. 2004). Cole et al. (2004) also reported that the number of undifferentiated epithelial cells increased in the fetal lung of GR null mice. These results suggest that the expression of functional GR may play an important role in making cells a more differentiated and less proliferative phenotype.

DOI: 10.1677/JME-08-0021

Online version via http://www.endocrinology-journals.org 
SIRPA (also known as SHPS-1, BIT, MFR, CD172a, or p84) is the first and best characterized member of the signal regulatory protein (SIRP) family and is a plasma membrane protein relatively ubiquitously expressed on myeloid cells, including macrophages (van Beek et al. 2005, Barclay \& Brown 2006). The cytoplasmic region of SIRPA contains four inhibitory immunoreceptor tyrosine-based inhibitory motifs which become phosphorylated upon ligand binding, and mediates recruitment and activation of tyrosine phosphatases of PTPN6 (SH2 domain-containing protein phosphastase-1) and PTPN11, which in turn, dephosphorylate specific protein substrates and thereby regulate cellular function (Veillette et al. 1998, Oshima et al. 2002, van Beek et al. 2005, Barclay \& Brown 2006). SIRPA plays important roles in immune regulation, generally in a negative fashion. It was also reported that the expression level of SIRPA decreased significantly in several types of tumor cells, including hepatocarcinoma cells, breast cancer cells, astrocytoma cells, glioblastoma cells, and most osteocarcinoma cells (Li et al. 1999, Ruhul Amin et al. 2002, Chen et al. 2004, Yan et al. 2004). Increasing SIRPA expression led to a reduction in tumor cell growth, migration, and cell transformation (Li et al. 1999, Ruhul Amin et al. 2002, Chen et al. 2004, Yan et al. 2004), suggesting that SIRPA is a negative regulator of cell growth. The signal transduction pathway involving the antiproliferative effect of SIRPA is unclear. Yan et al. (2004) reported that SIRPA negatively regulated MAPK3 activation in response to growth factors in human hepatocellular carcinoma cells. It was also reported that transfection with SIRPA plasmid decreased the expression of cyclinD1, a positive regulator of cell cycle, in hepatocarcinoma cells (Qin et al. 2005).

Cyclin/cyclin-dependent kinase (CDK) complexes are positive regulators in cell cycle, and CDKs activation induced by cyclins results in cells moving from one phase of the cell cycle to the next. CylinD1, cyclinB1, and CDK2 play pivotal roles in cell cycle, especially promoting G1/S phase transition (Kaldis \& Aleem 2005, Schwartz \& Shah 2005, Walker \& Assoian 2005). It has been reported that MAPKs play important roles in the regulation of cellular proliferation. The signals transmitted through this cascade, especially ERK1/2 and p38 can cause an activation of diverse molecules that regulate cell growth, survival, and differentiation (Robinson \& Cobb 1997, Garrington \& Johnson 1999).

The present study was designed to explore molecular mechanisms of antiproliferation of GC/GR by examining 1) the effect of GC/GR on the expression of SIRPA and its possible role in the antiproliferative effect of GC/GR in RAW264.7 cells and mouse peritoneal macrophage; 2) changes in the expression of CDK 2, cyclinD1, and cyclinB1 and the activity of MAPK1 and p38 in RAW-GR $(-)$ cells.

\section{Materials and methods}

\section{Materials}

Dex, mouse monoclonal antibodies against $\beta$-actin, and 3-(4,5 dimethylthiazol-2-yl)-2,5-diphenyl-2H tetrazolium bromide (MTT) were purchased from SigmaAldrich Chemicals. Rabbit polyclonal antibodies against SIRPA and cyclinD1 were obtained from StressGene and Neo Marker companies respectively. Rabbit polyclonal antibodies against GR, cyclin B1, ERK1/2, and phosphorylated ERK1/2 were from Santa Cruz Biotechnology (Santa Cruz, CA, USA). Antibodies against p38 and phosphorylated p38 were purchased from Cell Signaling Technology (Beverly, MA, USA). Goat antirabbit-AP and goat anti-mouse-AP conjugate were from Bio-Rad Laboratories. RPMI 1640 medium was obtained from Life Technologies Inc. BCA protein assay kit was purchased from Pierce (Rockford, IL, USA). SYBR Green was from OPEN company. The SIRPA expression vector (pcDNA3-SIRPA) was generously provided by Prof Wang (Yan et al. 2004).

\section{Cell lines and isolation of mouse peritoneal macrophages}

Murine macrophage cell line RAW264.7 was obtained from American Type Culture Collection (Rockville, MD, USA). RAW-GR (-)and RAW-control cells were established by stable transfection of RAW264.7 cells with GRsiRNA expression vector and nonspecific-siRNA vector respectively (Zhu et al. 2004). Mouse peritoneal exudates macrophages were harvested by peritoneal lavage from kunming mouse by i.p. injection of $1 \mathrm{ml}$ sterile ice-cold physiological saline five times. The cells were resuspended in RPMI 1640 and seeded in 96- or 24-well plate for $1.5 \mathrm{~h}$, the supernatant was then discarded and the cells cohered to the plate were mouse peritoneal macrophages (An et al. 2007).

\section{Cell culture}

RAW264.7, RAW-GR(-), and RAW-control cells were cultured in RPMI 1640 medium containing $10 \%$ heatinactivated fetal bovine serum (FBS) at $37^{\circ} \mathrm{C}$ in a humidified incubator of $5 \% \mathrm{CO}_{2}$. To examine the effect of Dex on the expression of SIRPA at the mRNA and protein levels, these cells were cultured in RPMI 1640 medium containing 10\% charcoal-dextran-treated FBS (CD-FBS) for $24 \mathrm{~h}$, and then treated with ethanol or different concentrations of Dex for the indicated times. Cortisol in CD-FBS was not detectable by RIA. Mouse peritoneal macrophages were cultured in RPMI 1640 medium containing 10\% CD-FBS and treated with ethanol or $10^{-7} \mathrm{M}$ Dex with or without $10^{-6} \mathrm{M}$ RU486 for 2 days. 


\section{Quantitative real-time PCR}

Total RNA was extracted with TRIzol reagent and $2 \mu \mathrm{g}$ total RNA was subjected to synthesizing first-strand cDNA by reverse transcription system (Promega) according to the manufacturer's instructions.

PCR primers used in this study are as follows: SIRPA, sense $5^{\prime}$-CTT TGC TCG TAG TCC TGC- $3^{\prime}$ and antisense $5^{\prime}$-CTG CGT ATG TGA TGT CGT-3' (177 bp); $\beta$-actin, sense 5'-CTG TAT GCC TCT GGT CGT AC-3' and antisense $5^{\prime}$-TGA TGT CAC GCA CGA TTT CC- $3^{\prime}$ (214 bp). SYBR Green was used as the fluorescence reagent. cDNA template (100 and $50 \mathrm{ng}$ for amplifying SIRPA and $\beta$-actin respectively) was added to the mixture of $2 \mu \mathrm{l} 10 \times$ buffer, $1.6 \mu \mathrm{l} 25 \mathrm{mM} \mathrm{MgCl}_{2}, 0.5 \mu \mathrm{l}$ $20 \mathrm{mM}$ dNTP, $0 \cdot 3 \mu 120 \mathrm{mM}$ primers, $1.5 \mathrm{U}$ Taq enzyme, and $1 \cdot 0 \mu \mathrm{l}$ of $20 \times \mathrm{SYBR}$ Green to a final volume of $20 \mu \mathrm{l}$. Amplification of SIRPA and $\beta$-actin was performed in different tubes, with the same process: 36 cycles of $15 \mathrm{~s}$ denaturation at $94^{\circ} \mathrm{C}, 20 \mathrm{~s}$ annealing at $57^{\circ} \mathrm{C}, 15 \mathrm{~s}$ elongation at $72{ }^{\circ} \mathrm{C}$, and a final extension for $10 \mathrm{~min}$ at $72{ }^{\circ} \mathrm{C}$. The mRNA ratio of Sirpa and actin was calculated as follows: we first drew the standard curves of SIRPA and $\beta$-actin by measuring the threshold cycles (CT) of standards of different concentrations, which had strong positive relationship with the start copy number (i.e. cDNA amounts) of a gene in linear amplification period. Then the cDNA amounts of SIRPA and $\beta$-actin of each testing sample were calculated according to their standard curves, and cDNA amount of SIRPA was normalized by the corresponding $\beta$-actin.

\section{Transient transfection of cells}

Cells were seeded in a 24 -well plate $\left(4 \times 10^{4}\right.$ cells/well $)$ or a 6 -well plate $\left(2 \times 10^{5}\right.$ cells/well) for $24 \mathrm{~h}$ and then transiently transfected with $1 \mu \mathrm{g}$ (for the 24-well plate) or $4 \mu \mathrm{g}$ (for the 6-well plate) of the control vector (pcDNA3) or SIRPA expression plasmid using LipofectAMINE Plus reagent (Invitrogen) according to the manufacturer's instructions.

\section{Western blot analysis}

Cells were lysed in ice-cold lysis buffer (Stamatakis $e t$ al. 2002; $10 \mathrm{mM}$ Tris $(\mathrm{pH} 7 \cdot 5), 0 \cdot 1 \mathrm{mM}$ EDTA, $0 \cdot 1 \mathrm{mM}$ EGTA, $0 \cdot 5 \%$ SDS, $0 \cdot 1 \mathrm{mM}$ mercaptoethanol, $2 \mu \mathrm{g} / \mathrm{ml}$ of each of the protease inhibitors leupeptin, aprotinin, and pepstatin) for $10 \mathrm{~min}$. The lysate was briefly sonicated on ice, and spun at $12893 \mathrm{~g}$ for $10 \mathrm{~min}$ to remove the insoluble material. Protein concentrations of the supernatant were measured with a BCA protein assay kit. Twenty to fifty micrograms of extracts were loaded on 10\% SDS-PAGE gel. After electrophoresis, the proteins were electrotransferred to nitrocellulose membranes, which were blocked with $5 \%$ nonfat milk, and probed overnight with antibodies against SIRPA (1:200), GR (1:1000), cyclinB1, cyclinD1, CDK2 (1:1000), MAPK1, p38 (1:500), or $\beta$-actin $(1: 10000)$. The membranes were then washed, exposed to alkaline phosphatase-conjugated secondary antibodies for $2 \mathrm{~h}$, and finally colored with phosphatase substrate 5-bromo-4-chloro-3-indolyl phosphate-nitro blue tetrazolium (BCIP-NBT).

The blots were quantified with Tanon software (Shanghai, China). In each experiment, we first normalized the levels of proteins interested in treated samples and control. We then compared the normalized numbers from three independent experiments. The average level from these experiments was calculated and the significance test performed.

\section{Cell proliferation assay}

Cell proliferation of RAW264.7, RAW-GR(-), and RAW-control cells was measured by MTT assay (Carmichael et al. 1987) or cytometry. Briefly, cells were seeded in duplicate at $3 \times 10^{4}$ cells per well in a 24 -well plate for MTT assay or $2 \times 10^{5}$ cells per well in a 6 -well plate for cytometry. After incubation for 2 days, cells in each well were collected and counted with a hemacytometer or examined by MTT assay. To each well, $200 \mu \mathrm{l}$ RPMI 1640 medium containing $5 \mathrm{mg} / \mathrm{ml}$ MTT (Sigma-Aldrich Chemicals) solution was added. Cells were incubated for $4 \mathrm{~h}$ at $37^{\circ} \mathrm{C}$, followed by solubilization with $200 \mu \mathrm{l}$ dimethyl sulfoxide (DMSO) solutions. Then the O.D. value was measured using a Bio-Rad (model 550) microplate reader at $550 \mathrm{~nm}$ with DMSO as blank. To ensure the reliability of MTT results, the linear response range of RAW264.7 cells between the results of MTT assay and the number of cells was explored. There was good linear correlation when RAW264.7 cells were seeded at the densities from $1 \times 10^{4}$ to $4 \times 10^{5}$ cells per well in a 24-well plate and cultured in medium for 4 days (data not shown).

The proliferation of mouse peritoneal macrophage was estimated by CCK-8 assay according to the manufacturer's guidelines. This assay is based on the cleavage of the tetrazolium salt WST- 8 by mitochondrial dehydrogenase in viable cells. Briefly, mouse peritoneal macrophages $\left(2 \times 10^{5}\right.$ cells/well $)$ were incubated with $100 \mu \mathrm{l}$ culture medium in 96-multiwell plates. After incubation with $10^{-7} \mathrm{M} \mathrm{GC}$ with or without $10^{-6} \mathrm{M}$ RU486 for 2 days, the media were removed and $100 \mu \mathrm{l}$ DMEM containing CCK-8 $(10 \mu \mathrm{l})$ was added to each well. After a further $1-\mathrm{h}$ incubation at $37^{\circ} \mathrm{C}$, the absorbance at $450 \mathrm{~nm}$ of each well was measured with a microplate reader. Each experiment was repeated thrice, and the data represent the mean of all measurements. 


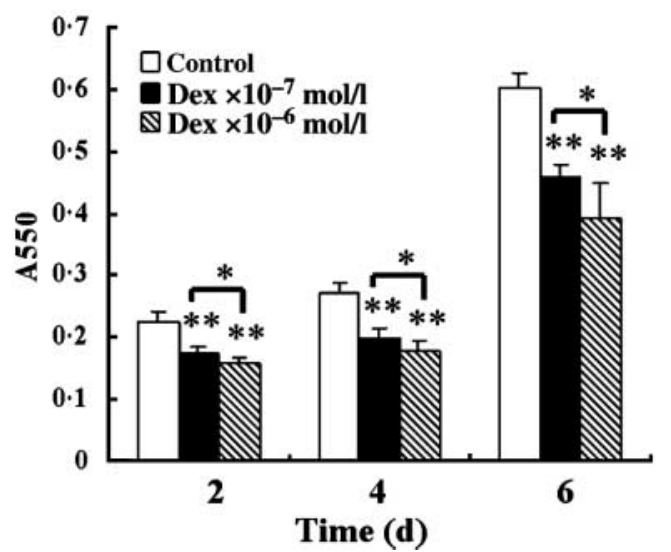

Figure 1 Dex inhibits growth of RAW264.7 cells in a dosedependent manner. RAW264.7 cells were seeded at $1 \times 10^{4}$ cells per well in a 24-well plate in duplicate and cultured in medium containing ethanol, $10^{-7} \mathrm{M}$ or $10^{-6} \mathrm{M}$ Dex for different periods of time. Proliferation of the cells was monitored by MTT assay. Values plotted are mean \pm S.D. of three independent experiments. ${ }^{\star} P<0.05$ between $10^{-7} \mathrm{M}$ and $10^{-6} \mathrm{M}$ Dex treatment, ${ }^{\star \star} P<0.01$ versus control.
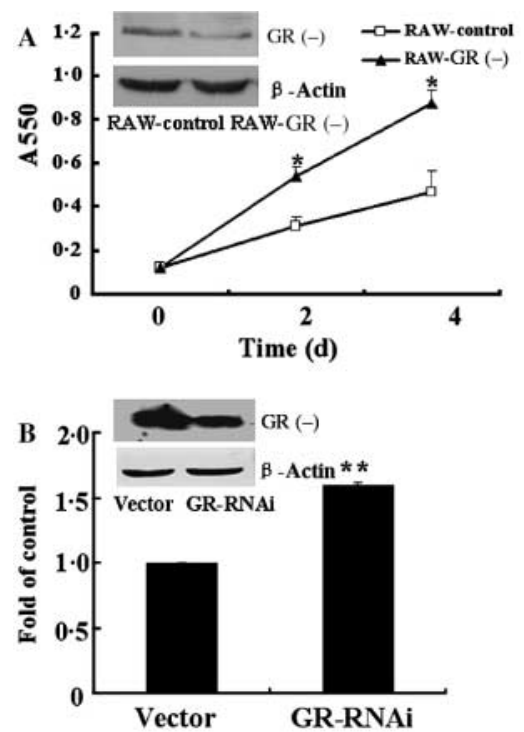

Figure 2 Inhibition of GR expression in RAW264.7 cells by stably or transiently transfected with GR RNA interference vectors promotes proliferation of the cells. Proliferation of RAW264.7 cells was assessed by MTT assay after stably transfected with (A) GR RNA interference vector pSilencer2.1-U6-GR (RAW-GR $(-)$ ) or (B) 4 days following transiently transfected with pSilencer2.1-U6GR. (mean \pm s.D., $n=3$ ), ${ }^{\star} P<0.05$ versus RAW-control cells (stably transfected with pSilencer2.1-U6-control), ${ }^{\star \star} P<0.01$ versus RAW264.7 cells transiently transfected with pSilencer2.1U6-control. GR expression was detected by western blot.

\section{RNAi of SIRPA}

Small interfering RNA (siRNA) of SIRPA was used to inhibit the expression of SIRPA in RAW264.7 cells by transient transfection with SIRPA siRNA oligonucleotide. Two SIRPA siRNA oligonucleotides designed by Genechem Co., (Shanghai, China) matched nonconserved 19 nucleotide sequences within the mouse Sirpa mRNA. The sequences of oligonucleotides used to create SIRPA RNAi were: 5'-CCC TGA CTA TCT GGT CTT A-3' $; 5^{\prime}$-GCC TGA CAC AGA AAT ACA A-3' . They expressed a hairpin siRNA with limited homology to any known sequences in human, mouse, and rat genomes. The oligonucleotides were annealed before transient transfection of RAW264.7 cells. The siRNA control was provided by the same company.

\section{A Real time PCR}

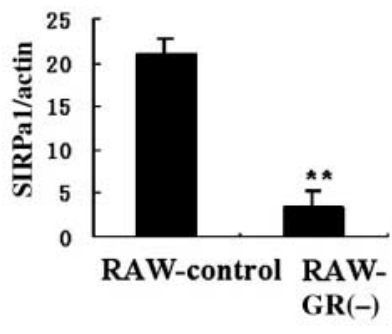

B Western blot

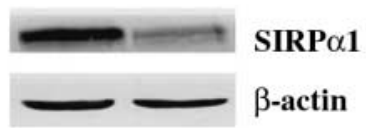

RAW-control RAW-GR(-)

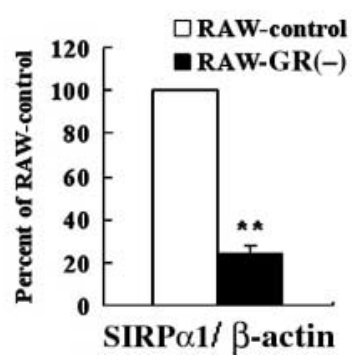

Figure 3 Inhibition of GR expression in RAW264.7 cells by RNA interference decreases SIRPA expression at both $\mathrm{mRNA}$ and protein levels. (A) Sirpa mRNA expression in RAW-GR(-) and RAW-control cells was detected by real-time PCR (mean \pm s.D., $n=3$ ), ${ }^{\star *} P<0.01$ versus RAW-control. (B) Protein levels of SIRPA in RAW-GR $(-)$ and RAW-control cells were examined by western blot analysis. Similar results were obtained from three independent experiments. Using Tanon software (Shanghai, China), the blots of SIRPA in these two cells were quantified and normalized against $\beta$-actin. Then the normalized numbers from three independent experiments were compared. The values are expressed as percent of the level of RAW-control, as shown in the histogram of (B). (mean \pm s.D., $n=3$ ), ${ }^{\star \star} P<0.01$ versus RAW-control. 


\section{Statistical analysis}

Data are expressed as mean \pm s.D. of the separate experiments. Statistical significance was determined by Student's $t$-test with a value of $P<0.05$ considered as statistically significant.

\section{Results}

\section{GC/GR negatively regulates the proliferation of RAW264.7 cells}

The effect of Dex on RAW264.7 cell growth was examined by MTT assay. Figure 1 shows that the growth of RAW264.7 cells was inhibited by Dex in a concentration-dependent manner. About 27 and 34\% inhibition of cell proliferation was observed at day 4 after treatment with $10^{-7}$ and $10^{-6} \mathrm{M}$ Dex respectively, and no further increase of inhibition rate was observed at day 6. Furthermore, when RAW264.7 cells were stably transfected with GR siRNA expression vector, the expression of GR was significantly decreased, accompanied by an increase in cell proliferation (Fig. 2A). The number of RAW-GR $(-)$ cells was almost twofold of that of the RAW-control cells after 4-day culture. The proliferation promotion by GR knockdown was further confirmed in transiently transfection assay (Fig. 2B). These results indicate that GC/GR negatively regulates the growth of RAW264.7 cell.

\section{GC/GR induces the expression of SIRPA at mRNA and protein levels in RAW264.7 cells}

Although GR has been proved to play an important role in inhibiting cell proliferation, only a few target genes that mediate antiproliferative effect of GR/GC on macrophages are known. Considering that SIRPA is highly expressed in macrophages and inhibits the proliferation of different cell-type-derived tumor cells, we presumed that SIRPA may be involved in the

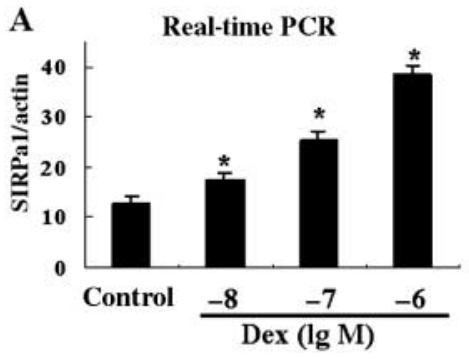

B
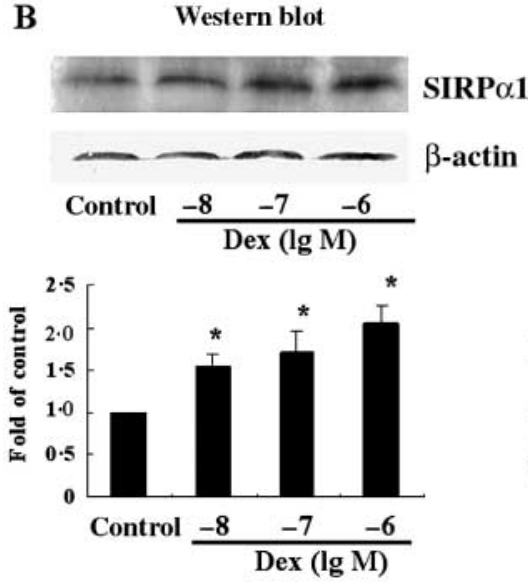

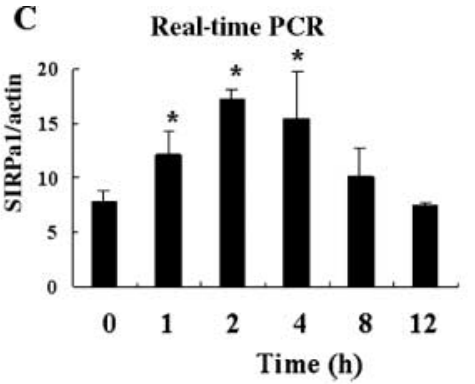

D Western blot
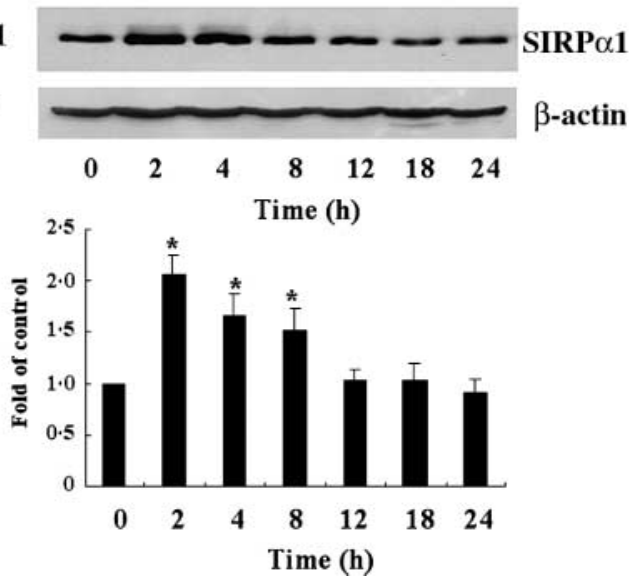

Figure 4 Dex increases SIRPA expression in a dose- and time-dependent manner in RAW264.7 cells. (A and C) Sirpa mRNA expression in RAW264.7 cells after treatment with different concentrations of Dex and with $10^{-7} \mathrm{M}$ Dex for different time was examined by real-time PCR, (mean \pm s.D., $n=3$ ), ${ }^{\star} P<0.05$ versus control. (B and D) Protein level of SIRPA in RAW264.7 cells was examined by western blot analysis. Similar results were obtained from three independent experiments. The blots of SIRPA were quantified by Tanon software and normalized against $\beta$-actin. Then the normalized numbers from three independent experiments were compared. The values are expressed as fold of the level of control, as shown in the histograms of $\mathrm{B}$ and $\mathrm{D}$. (mean \pm s.D., $n=3$ ), ${ }^{*} P<0.05$ versus control. 
antiproliferative characteristic of GC/GR. To this end, the expression of SIRPA in RAW-GR $(-)$ cells was then examined by quantitative real-time PCR and western blot analysis. The results showed that mRNA and protein levels of SIRPA in RAW-GR $(-)$ cells reduced to about 16 and $24 \%$ of RAW-control cells respectively (Fig. 3).

To determine whether GC up-regulated the expression of SIRPA, we further investigated the mRNA and protein levels of SIRPA in RAW264.7 cells treated with different concentrations of Dex or with $10^{-7} \mathrm{M}$ Dex for different times by quantitative real-time PCR and western blot respectively. Figure 4 shows that Dex induced the expression of SIRPA at mRNA and protein levels in dose- and time-dependent manner. The mRNA level of Sirpa significantly increased $1 \mathrm{~h}$
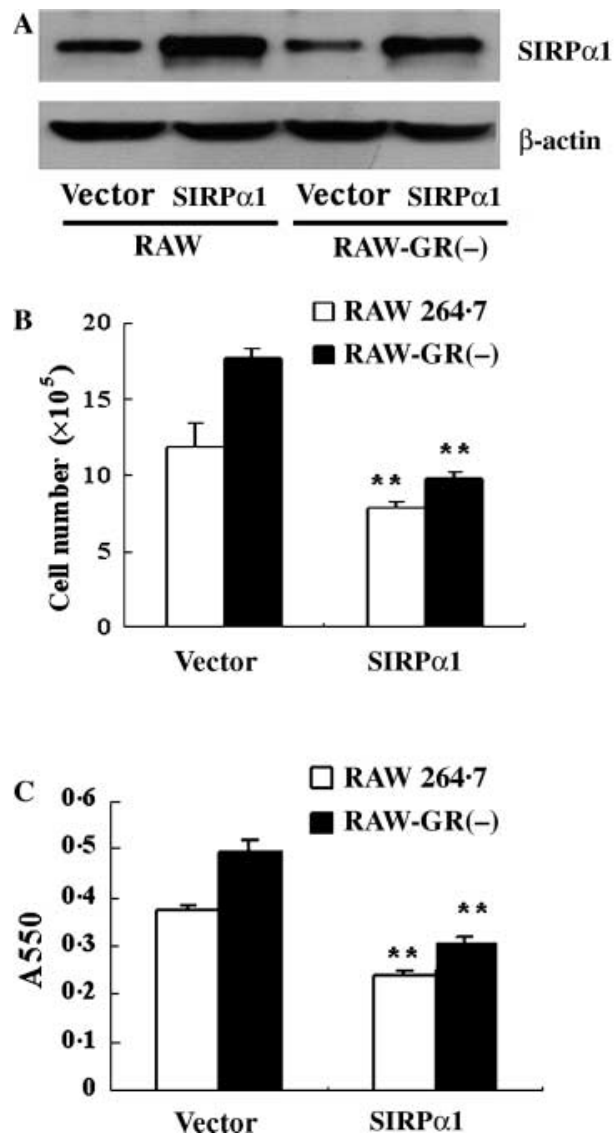

Figure 5 Transfection of RAW-GR(-) and RAW264.7 cells with SIRPA expression plasmid inhibits cell proliferation. (A) RAW$\mathrm{GR}(-)$ and RAW264.7 cells were transfected with SIRPA plasmid or control vector for $24 \mathrm{~h}$ and the protein levels of SIRPA was detected by western blot analysis. These blots are representative of three independent experiments. After transfection with SIRPA vector or control vector, cells were seeded and cultured in duplicate for 2 days in $(\mathrm{B})$ a 6 -well $\left(2 \times 10^{5}\right.$ cells/well) plate for cytometry or $(C)$ a 24 -well plate $\left(3 \times 10^{4}\right.$ cells/well) for MTT assay. (mean \pm S.D., $n=3$ ), ${ }^{\star \star} P<0.01$ versus cells transfected with control vector, $n=3$. after treatment with $10^{-7} \mathrm{M}$ Dex, with the maximal induction effect at $2 \mathrm{~h}(2 \cdot 2$-fold of the control, $P<0 \cdot 05$; Fig. 4C). A similar increase in SIRPA protein expression was also observed following the incubation of cells with $10^{-7}$ M Dex $(P<0 \cdot 05$; Fig. 4D).

\section{SIRPA signaling is involved in GC/GR-mediated inhibition of RAW264.7 cell proliferation}

As the expression of SIRPA was regulated by GC/GR in RAW264.7 cells and increased proliferation was associated with a significant decrease in the expression of SIRPA in RAW-GR $(-)$ cells, we hypothesized that SIRPA might be involved in the regulation of RAW264.7 cell growth by GC/GR, and therefore we examined the effect of SIRPA on cell proliferation by MTT assay and cytometry. It was found that the expression of SIRPA in RAW-GR $(-)$ cells and in RAW264.7 cells transiently transfected with SIRPA plasmids increased by about $2 \cdot 5$-fold (Fig. 5A), which resulted in about 40 and $34 \%$ inhibition of proliferation in RAW-GR $(-)$ cells and in RAW264.7 cells respectively (Fig. 5B and C). Then we further examined the role of SIRPA in GC/GRinduced proliferation inhibition of RAW264.7 cells by inhibiting the expression of SIRPA using small interference RNA. As shown in Fig. 6A, the level of

$\mathbf{A}$
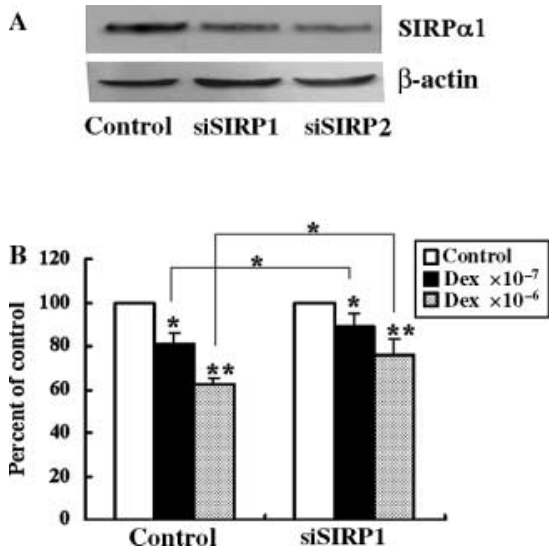

Figure 6 Inhibition of SIRPA expression in RAW264.7 cells by RNA interference attenuates Dex-induced inhibition of cell proliferation. (A) RAW264.7 cells were transfected with small interference RNA (siRNA) for SIRPA or siRNA control and the protein levels of SIRPA in the cells were detected by western blot analysis. These blots are representative of three independent experiments. After transfection, RAW264.7 cells were seeded at $1 \times 10^{4}$ cells per well in a 24 -well plate in duplicate and cultured in medium containing ethanol, $10^{-7} \mathrm{M}$ or $10^{-6} \mathrm{M}$ dexamethasone (Dex) for 4 days. (B) Proliferation of the cells was monitored by MTT assay. The values of A550 values were normalized against corresponding blank control to show clearer the difference of inhibition rate. Then the normalized numbers from three independent experiments were compared. The values are expressed as percent of the level of corresponding control. ${ }^{\star \star} P<0.01$ versus control, ${ }^{\star} P<0.05$ versus control. 
SIRPA protein in siSIRP 1 and siSIRP 2 cells reduced to 29 and $31 \%$ of that in cells treated with control siRNA respectively. The inhibition of SIRPA expression attenuated Dex-induced inhibition of cell proliferation in siSIRP 1 cells as compared with that in control cells (Fig. 6B), indicating that SIRPA negatively regulates the proliferation of RAW264.7 cells and SIRPA signaling is involved in the inhibitory effect of GC/GR on the proliferation of these cells.

\section{SIRPA signaling is also associated with GC/GR- mediated inhibition of mouse peritoneal macrophage proliferation}

Does the proliferation inhibition effect of Dex on RAW264.7 cells exist in primary mouse peritoneal macrophages? Is the proliferation inhibition effect of Dex on primary mouse peritoneal macrophages also associated with SIRPA? To this end, we examined the effect of Dex on the proliferation of mouse peritoneal macrophages by CCK- 8 assay and found that cell growth was decreased obviously two days after treatment with $10^{-7}$ mol Dex and the inhibition of proliferation was completely reversed by GR antagonist, RU486, while RU486 itself had no effect on cell proliferation (Fig. 7A). At the same time, we detected the expression of mRNA of Sirpa by real-time PCR and found that the expression of SIRPA was increased remarkably by treatment with $10^{-7} \mathrm{M}$ Dex and RU486 partially inhibited the enhancement of SIRPA induced by GC, while RU486 itself had no effect on the expression of SIRPA (Fig. 7B). These results indicated that GC also inhibited the growth of primary mouse peritoneal macrophage through GR mediation and the inhibitory effect of GC/GR was also associated with SIRPA.

\section{Increased expression of CDK2, cyclinD1, and cyclinB1 but not ERK1/2 and p38 phosphorylation may be associated with the increased proliferation and cell cycle change of RAW-GR( - ) cells}

Previous studies demonstrated that GCs inhibited cell growth by lengthening the cell cycle, mainly through arresting cells in the G1 phase (Funakoshi et al. 2005, Sundberg et al. 2006). The decreased percentage of G1 phase cells and the increased percentage of $S$ phase cells were found in RAW-GR $(-)$ cells as compared with that of RAW-control cells (Fig. 8A). CDK2, cylinD1, and cyclinB1 play pivotal roles in cell cycle, especially promoting G1/S phase transition (Kaldis \& Aleem 2005, Schwartz \& Shah 2005, Walker \& Assoian 2005), so we detected the expression of these proteins in RAW-GR $(-)$ cells. Figure 8B shows that the expression of CDK2, cyclinD1, and cyclinB1 proteins increased
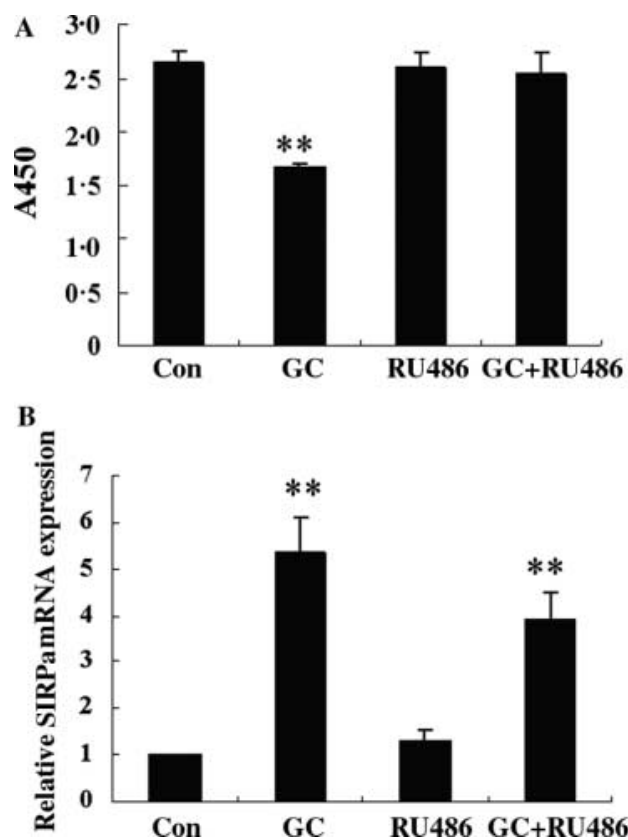

Figure 7 Increase in Sirpa mRNA induced by Dex in mouse peritoneal macrophages is associated with the proliferation inhibition of Dex on the cells. Mouse peritoneal macrophages were isolated and seeded at $4 \times 10^{5}$ cells per well in a 96-well plate in duplicate and cultured in medium containing ethanol, $10^{-7} \mathrm{M}$ Dex with or without GR antagonist $10^{-6}$ M RU486 for 2 days. (A) Proliferation of the cells was monitored by CCK-8 assay. Values plotted are mean \pm S.D. of three independent experiments. ${ }^{\star \star} P<0.01$ versus control. Mouse peritoneal macrophages were isolated and seeded at $2 \times 10^{6}$ cells per well in a 24 -well plate and treated with $10^{-7} \mathrm{M}$ Dex with or without $10^{-6} \mathrm{M}$ RU486, then Sirpa mRNA expression in the cells was examined by real-time PCR (B) (mean \pm s.D., $n=3$ ), ${ }^{\star \star} P<0.01$ versus control.

significantly in RAW-GR $(-)$ cells by about $2 \cdot 1-, 1 \cdot 7-$, and $2 \cdot 2$-fold respectively, as compared with that in RAWcontrol cells, suggesting that these positive regulators of cell cycle may be associated with faster growth resulted from GR knocking down.

In recent years, evidence has emerged that GR plays important roles in regulating cell proliferation by modulating the activity of ERK1/2 and p38 (Miller et al. 2005, Qin et al. 2005), so we examined the activated status of these kinases in RAW-GR $(-)$ and RAW-control cells by western blot. We failed to find significant difference in phosphorylated-ERK1/2 or -p38 level between RAW-GR $(-)$ and RAW-control cells (Fig. 9).

\section{Discussion}

GC effectively suppresses immune and inflammatory responses and inhibits the growth of several types of cells, but the role of GC and its receptor on macrophage proliferation is unclear. In our previous work, we found that RAW-GR $(-)$ cells stably 

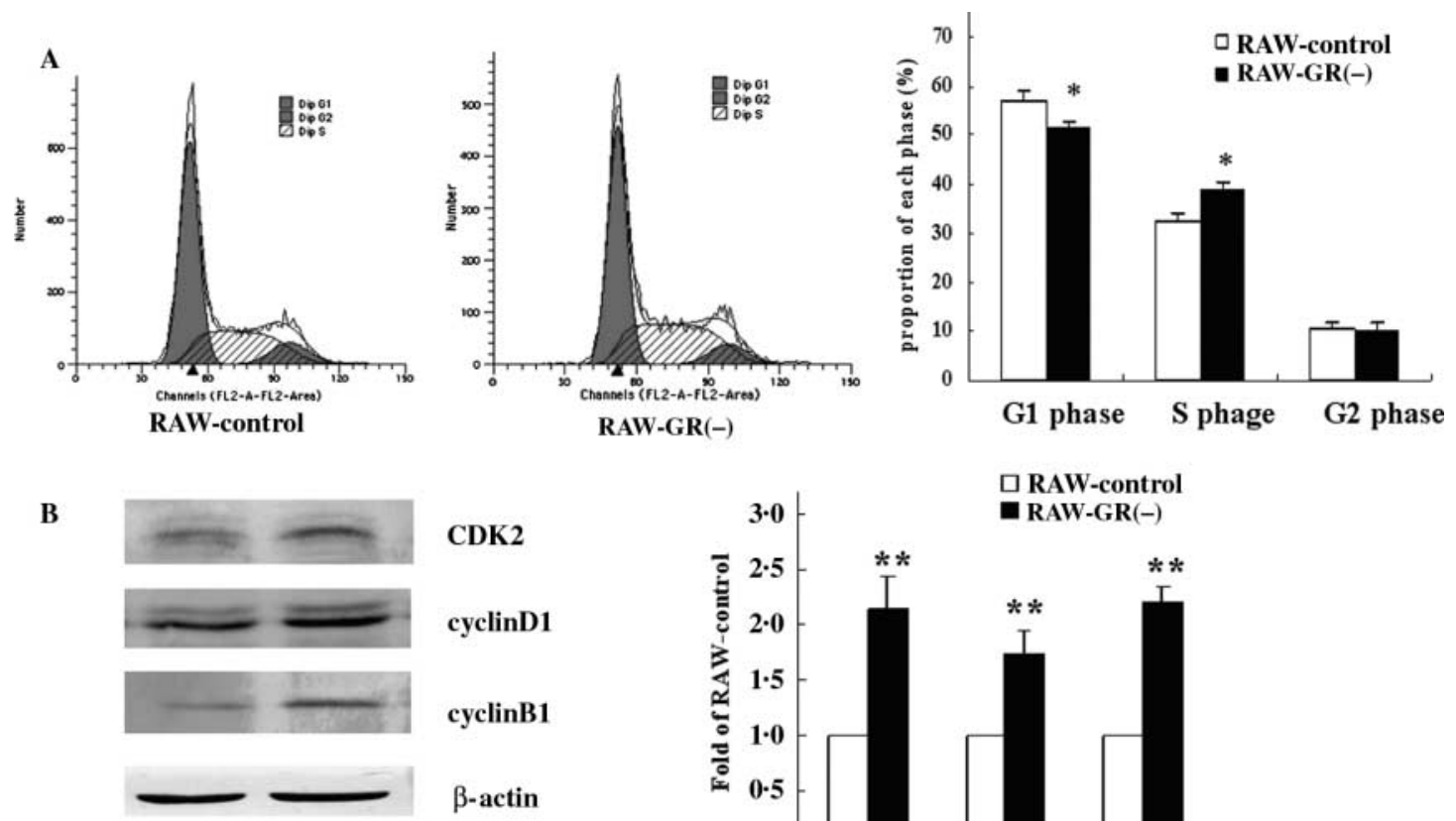

RAW-control RAW-GR(-)

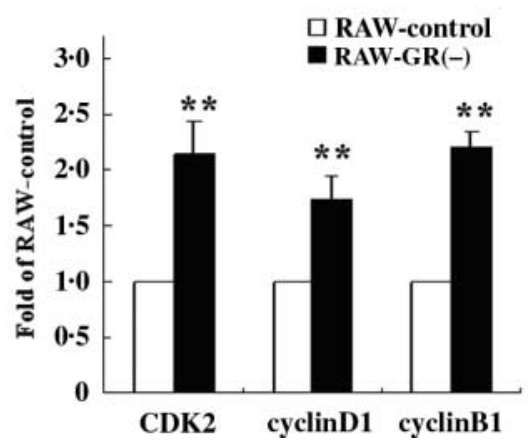

Figure 8 Increase in CDK2, cyclinD1, and cyclinB1 in RAW-GR(-) cells may be associated with the increased proliferation and cell cycle change of RAW-GR (-). (A) Change of cell cycle in RAW-GR(-) cells as compared with RAW-control cells. The RAW-GR(-) and RAWcontrol cells were cultured for $12 \mathrm{~h}$ with $10 \%$ fetal bovine serum followed by $24 \mathrm{~h}$ starving with serum-free medium. The cells were collected to determine the cell percentage of G1, G2, and S phases by FACSCalibur flow cytometer. Values shown are expressed as proportion of each phase and are the mean \pm S.D. of three independent experiments, as shown in the histogram. ${ }^{\star} P<0.05$ versus RAWcontrol. (B) The protein levels of CDK2, cyclinD1, and cyclinB1 in RAW-GR $(-)$ and RAW-control cells were examined by western blot analysis. These blots are representative of three independent experiments. The blots of CDK2, cyclinD1, and cyclinB1 in these two cells were quantified by Tanon software (Shanghai, China) and normalized against $\beta$-actin. Then the normalized numbers from three independent experiments were compared. The values of expression levels are expressed as fold of the level of RAW-control, as shown in the histogram. (mean \pm s.D., $n=3$ ), ${ }^{\star \star} P<0.01$ versus RAW-control.

transfected RAW264.7 cells with GR-siRNA expression vector grew faster by about twofold (Zhu et al. 2004). A similar result was obtained by transient transfection with GR RNAi plasmid in this study. While treatment with $10^{-7}$ M Dex for 4 days resulted in only $27 \%$ proliferation inhibition of RAW264.7 cells. More important, the proliferation of RAW-GR $(-)$ cells shows no significant difference between cultured in RPMI 1640 medium containing 10\% heat-inactivated FBS and $10 \%$ charcoal-stripped serum. The inhibition effect of GC/GR on cell proliferation was also found in primary mouse peritoneal macrophages. These results indicated that GR negatively regulated the growth of RAW264.7 cells and mouse peritoneal macrophages, and the inhibitory effect of GR may work mainly in a GC-independent manner. Several studies on hormonesensitive carcinomas in vitro and in vivo in nude mice also showed similar results in that loss of expression of functional steroid receptors was often associated with the development of less differentiated tumors with a consequently poor prognosis (Baldi et al. 2003, Nightingale et al. 2003, Litvinov et al. 2004). For example, the growth of prostate cancer cell lines lacking androgen receptor (e.g. PC-3 cells) was faster than that of cells expressing androgen receptors (e.g. LNCap cells) (Chlenski et al. 2001), and expressing transfected androgen receptor in PC-3 cells decreased the proliferation rate and cloning efficiency and induced a more differentiated phenotype (Yuan et al. 1993). Similar increased proliferation also found in some breast cancer cell lines that lack estrogen receptors (Platet $e t$ $a l$. 2004). These phenomena found in our laboratory and by other researchers indicate that expression of functional GR and other steroid receptors is very important for cells to become a more differentiated and less proliferative phenotype.

As mentioned in introduction, SIRPA is highly expressed in macrophages, and plays important roles in immune regulation and inhibits the proliferation of several types of cells. So we wondered whether the expression of SIRPA was regulated by GC/GR and whether there was a correlation between SIRPA expression and cell proliferation in RAW264.7 cells and mouse peritoneal macrophages. The expression of SIRPA was induced by Dex time and dose dependently in RAW264.7 cells, and decreased markedly in RAW- 
$\mathbf{A}$
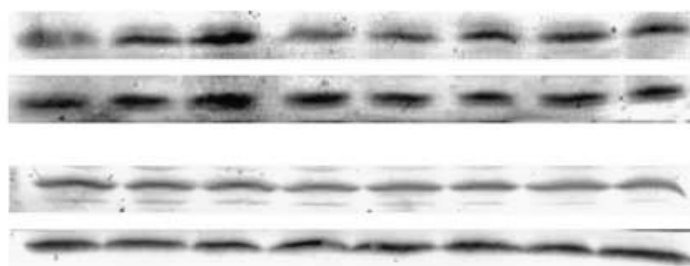

$0 \mathrm{~min} 5 \mathrm{~min} 20 \mathrm{~min} 60 \mathrm{~min}$

RAW-control

$0 \mathrm{~min} 5 \mathrm{~min} 20 \mathrm{~min} 60 \mathrm{~min}$
p-ERK1/2

Total ERK1/2

p-ERK1/2

Total ERK1/2
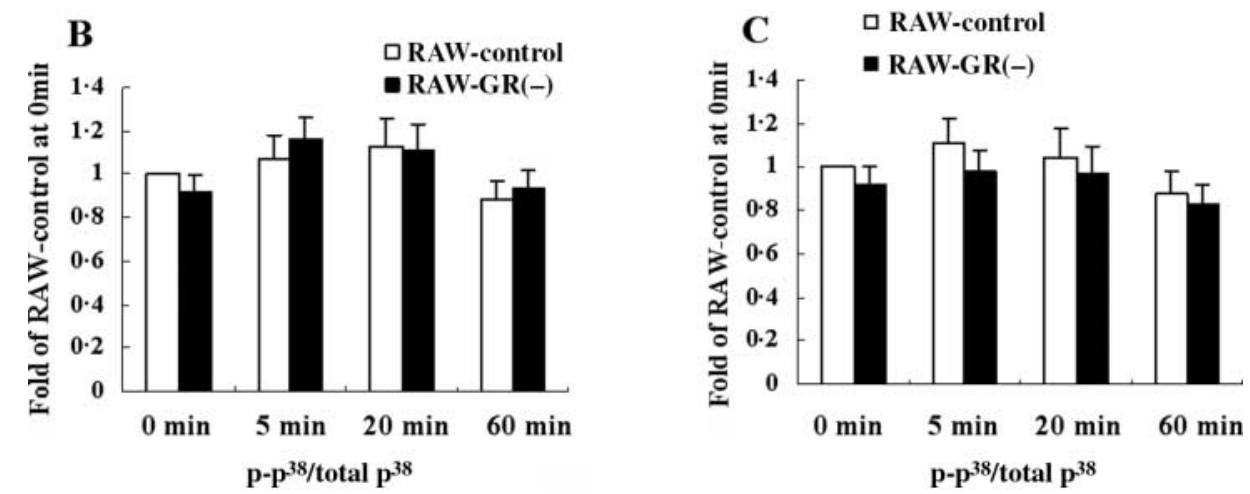

Figure 9 No change of protein levels of phosphorylated p38 and ERK1/2 is found in RAW-GR $(-)$ cells compared with RAW-control cells. (A) RAW-GR $(-)$ and RAW-control cells were starved with serum-free medium for $24 \mathrm{~h}$, and then recovered to be cultured with $10 \%$ fatal bovine serum for 5,20 , or $60 \mathrm{~min}$. The cells were collected to determine the activated status of p38 and ERK1/2 at the indicated above time with the antibody of anti-phosphorylated p38 and ERK1/2 by western blot analysis. The total p38 and ERK1/2 were used as the quantitative standard. (B) The ratio of $p-p 38$ and total p38 was expressed as fold of that of RAW-control cells at 0 min. There was no obvious difference between RAW-GR $(-)$ and RAW-control cells at three time points. Values shown are the mean \pm s.D. of three independent experiments. (C) The ratio of p-MAPK1/total MAPK1 was expressed as fold of the level of RAW-control at 0 min, and values shown are the mean \pm S.D. of three independent experiments.

GR $(-)$ cells. Overexpression of SIRPA in RAW-GR $(-)$ and RAW264.7 cells transiently transfected with SIRPA expression vector obviously inhibited the proliferation of these cells, while knockdown of SIRPA expression by siRNA attenuated Dex-induced inhibition of cell proliferation. In addition, the proliferation inhibition of GC/GR on mouse peritoneal macrophages was also associated with Dex-induced enhancement of SIRPA. These results support our hypothesis that SIRPA is a negative regulator of the proliferation in RAW264.7 cells and mouse peritoneal macrophages, and SIRPA signaling is involved in antiproliferative effect of GC/GR on these cells.

It is well known that GR up-regulates gene transcription by direct binding with GRE located in the promoter region of target genes (Dondi et al. 2001, Kudawara et al. 2001, Frankfurt \& Rosen 2004). We analyzed the sequence at the promoter region of Sirpa gene, and found no consensus GRE, but just several potential GRE half-sites (data not shown). It is not known whether these GRE half-sites could mediate the effect of Dex on SIRPA transcription. In addition to binding with GREs, GR can indirectly regulate gene expression by interacting with other classes of transcription factors, such as NF-אB, AP-1(Fos/Jun), TRP53 to exert its antiproliferative role (Crochemore et al. 2002, Sengupta \& Wasylyk 2004, Bladh et al. 2005). Whether these molecules are involved in GR-mediated SIRPA regulation remains to be investigated.

GC/GR has been known to play an antiproliferative role through inducing cell cycle arrest and regulating the expression of regulatory proteins of cell cycle in several types of cells, including osteosarcoma cells, hepatoma cells, and embryonic neural stem cells (Kudawara et al. 2001, Yamamoto et al. 2002, Frankfurt \& Rosen 2004, Sundberg et al. 2006). We had examined the protein levels of the cyclin-dependent kinase inhibitors (CDKi) CDKN1A and CDKN1B, and found that the expression of CDKN1B, but not CDKN1A was reduced in RAW-GR(-) cells (Zhu et al. 2004). In this study, we further demonstrated that increased proliferation of RAW-GR $(-)$ cells was associated with 
significant increase in positive regulatory proteins of cell cycle (CDK2, cyclinD1, and cyclinB1). These results indicate that GR plays a crucial role in cell cycle arrest through regulating the expression of regulatory proteins of cell cycle in macrophage cells.

ERK1/2 and p38 are also involved in the control of cell proliferation. Whether ERK1/2 and p38 are involved in GR knockdown-induced pro-proliferation process remains unknown. Our results showed no change in phosphorylated ERK1/2 and p38 expression, suggesting that ERK1/2 and p38 pathways did not participate in the regulation of GR-knockdown-sensitized proliferation in RAW264.7 cells.

In summary, in this study, we first demonstrated that GC/GR inhibited the proliferation of both established RAW264.7 cell line and mouse peritoneal macrophage. The novel evidence is provided that SIRPA, a negative modulator of cell growth induced by $\mathrm{GC} / \mathrm{GR}$, is involved in the antiproliferative effect of GC/GR on macrophages. Furthermore, the increase in CDK2, cyclinD1, and cyclinB1 may be also related to the increased proliferation of RAW-GR $(-)$ cells.

\section{Declaration of interest}

The authors declare that there is no conflict of interest that could be perceived as prejudicing the impartiality of the research reported.

\section{Funding}

This work was supported by grant from the National Natural Science Foundation of China (No.30300128).

\section{Acknowledgements}

We greatly appreciate the gift of SIRPA expression vector from Prof $\mathrm{H}$ Wang (International cooperative laboratory on signal transduction, Eastern Hepatobiliary Surgery Institute, China).

\section{References}

An P, Zhu JY, Yang Y, Lv P, Tian YH, Chen MK \& Luo HS 2007 KN-93, a specific inhibitor of CaMKII inhibits human hepatic stellate cell proliferation in vitro. World Journal of Gastroenterology 13 1445-1448.

Baldi E, Bonaccorsi L \& Forti G 2003 Androgen receptor: good guy or bad guy in prostate cancer invasion? Endocrinology 144 1653-1655.

Barclay AN \& Brown MH 2006 The SIRP family of receptors and immune regulation. Nature Reviews. Immunology 6 457-464.

van Beek EM, Cochrane F, Barclay AN \& van den Berg TK 2005 Signal regulatory proteins in the immune system. Journal of Immunology 175 7781-7787.

Bladh LG, Liden J, Dahlman-Wright K, Reimers M, Nilsson S \& Okret S 2005 Identification of endogenous glucocorticoid repressed genes differentially regulated by a glucocorticoid receptor mutant able to separate between nuclear factor-kappaB and activator protein-1 repression. Molecular Pharmacology 67 815-826.
Brummer E, Choi JH \& Stevens DA 2005 Interaction between conidia, lung macrophages, immunosuppressants, proinflammatory cytokines and transcriptional regulation. Medical Mycology 43 (Suppl 1) S177-S179.

Carmichael J, DeGraff WG, Gazdar AF, Minna JD \& Mitchell JB 1987 Evaluation of a tetrazolium-based semiautomated colorimetric assay: assessment of chemosensitivity testing. Cancer Research $\mathbf{4 7}$ 936-942.

Chen, TT, Brown EJ, Huang EJ \& Seaman WE 2004 Expression and activation of signal regulatory protein alpha on astrocytomas. Cancer Research 64 117-127.

Chlenski A, Nakashiro K, Ketels KV, Korovaitseva GI \& Oyasu R 2001 Androgen receptor expression in androgen-independent prostate cancer cell lines. Prostate 47 66-75.

Cole TJ, Solomon NM, Van Driel R, Monk JA, Bird D, Richardson SJ, Dilley RJ \& Hooper SB 2004 Altered epithelial cell proportions in the fetal lung of glucocorticoid receptor null mice. American Journal of Respiratory Cell and Molecular Biology 30 613-619.

Crochemore C, Michaelidis TM, Fischer D, Loeffler JP \& Almeida OF 2002 Enhancement of p53 activity and inhibition of neural cell proliferation by glucocorticoid receptor activation. FASEB Journal 16 761-770.

Distelhorst CW 2002 Recent insights into the mechanism of glucocorticosteroid-induced apoptosis. Cell Death and Differentiation 9 6-19.

Dondi D, Maggi R, Scaccianoce E, Martini L, Motta M \& Poletti A 2001 Expression and role of functional glucocorticoid receptors in the human androgen-independent prostate cancer cell line, DU145. Journal of Molecular Endocrinology 26 185-191.

Frankfurt O \& Rosen ST 2004 Mechanisms of glucocorticoid-induced apoptosis in hematologic malignancies: updates. Current Opinion in Oncology 16 553-563.

Funakoshi Y, Shiono H, Inoue M, Kadota Y, Ohta M, Matsuda H, Okumura M \& Eimoto T 2005 Glucocorticoids induce G1 cell cycle arrest in human neoplastic thymic epithelial cells. Journal of Cancer Research and Clinical Oncology 131 314-322.

Garrington TP \& Johnson GL 1999 Organization and regulation of mitogen-activated protein kinase signaling pathway. Current Opinion in Cell Biology 11 211-218.

Heitzer MD, Wolf IM, Sanchez ER, Witchel SF \& DeFranco DB 2007 Glucocorticoid receptor physiology. Reviews in Endocrine and Metabolic Disorders 8 321-330.

Kaldis P \& Aleem E 2005 Cell cycle sibling rivalry: Cdc2 vs. Cdk2. Cell Cycle 4 1491-1494.

Kudawara I, Ueda T, Yoshikawa H, Miyama T, Yamamoto T \& Nishizawa Y 2001 In vivo inhibition of tumour growth by dexamethasone in murine osteosarcomas. European Journal of Cancer 37 1703-1708.

Li B, Wang H \& Chen Z 1999 The association between signalregulatory protein alpha and hepatocellular carcinoma. Zhonghua Yi Xue Za Zhi 79 268-270.

Litvinov IV, Antony L \& Isaacs JT 2004 Molecular characterization of an improved vector for evaluation of the tumor suppressor versus oncogene abilities of the androgen receptor. Prostate 61 299-304.

Lloberas J, Soler C \& Celada A 1998 Dexamethasone enhances macrophage colony stimulating factor- and granulocyte macrophage colony stimulating factor-stimulated proliferation of bone marrow-derived macrophages. International Immunology 10 593-599.

Miller AL, Webb MS, Copik AJ, Wang Y, Johnson BH, Kumar R \& Thompson EB 2005 p38 Mitogen-activated protein kinase (MAPK) is a key mediator in glucocorticoid-induced apoptosis of lymphoid cells: correlation between p38 MAPK activation and site-specific phosphorylation of the human glucocorticoid receptor at serine 211. Molecular Endocrinology 19 1569-1583.

Nightingale J, Chaudhary KS, Abel PD, Stubbs AP, Romanska HM, Mitchell SE, Stamp GW, Lalani N et al. 2003 Ligand activation of the androgen receptor downregulates E-cadherin-mediated cell adhesion and promotes apoptosis of prostatic cancer cells. Neoplasia 5 347-361.

Oshima K, Ruhul Amin AR, Suzuki A, Hamaguchi M \& Matsuda S 2002 SHPS-1, a multifunctional transmembrane glycoprotein. FEBS Letters 519 1-7. 
Pagniello KB, Bols NC \& Lee LE 2002 Effect of corticosteroids on viability and proliferation of the rainbow trout monocyte/macrophage cell line, RTS11. Fish and Shellfish Immunology 13 199-214.

Platet N, Cathiard AM, Gleizes M \& Garcia M 2004 Estrogens and their receptors in breast cancer progression: a dual role in cancer proliferation and invasion. Critical Review in Oncology and Hematology 51 55-67.

Qin JM, Yan HX, Wan XW, Liu SQ, Zeng JZ, Cao HF, Wu MC \& Wang HY 2005 Effects of signal regulatory proteinalphal on proliferation of hepatocellular carcinoma: a preliminary study. Hepatobiliary and Pancreatic Diseases International 4 244-248.

Robinson MJ \& Cobb MH 1997 Mitogen-activated protein kinase pathways. Current Opinion in Cell Biology 9 180-186.

Ruhul Amin AR, Machida K, Oshima K, Oo ML, Thant AA, Senga T, Matsuda S, Akhand AA, Maeda A, Kurosaki T \& Hamaguchi M 2002 A role for SHPS-1/SIRPalphal in IL-1beta- and TNFalphadependent signaling. Oncogene 21 8871-8878.

Schwartz GK \& Shah MA 2005 Targeting the cell cycle: a new approach to cancer therapy. Journal of Clinical Oncology 23 9408-9421.

Sengupta S \& Wasylyk B 2004 Physiological and pathological consequences of the interactions of the p53 tumor suppressor with the glucocorticoid, androgen, and estrogen receptors. Annals of the New York Academy of Sciences 1024 54-71.

Stamatakis K, Hernandez-Perera O \& Perez-Sala D 2002 Isoprenylation of RhoB is necessary for its degradation. A novel determinant in the complex regulation of RhoB expression by the mevalonate pathway. Journal of Biological Chemistry 277 49389-49396.

Sundberg M, Savola S, Hienola A, Korhonen L \& Lindholm D 2006 Glucocorticoid hormones decrease proliferation of embryonic neural stem cells through ubiquitin-mediated degradation of cyclin D1. Journal of Neuroscience 26 5402-5410.
Veillette A, Thibaudeau E \& Latour S 1998 High expression of inhibitory receptor SHPS-1 and its association with protein-tyrosine phosphatase SHP-1 in macrophages. Journal of Biological Chemistry 273 22719-22728.

Walker JL \& Assoian RK 2005 Integrin-dependent signal transduction regulating cyclin D1 expression and G1 phase cell cycle progression. Cancer Metastasis Reviews 24 383-393.

Yamamoto T, Nishiguchi M, Inoue N, Goto HG, Kudawara I, Ueda T, Yoshikawa H, Tanigaki Y \& Nishizawa Y 2002 Inhibition of murine osteosarcoma cell proliferation by glucocorticoid. Anticancer Research 22 4151-4156.

Yan HX, Wang HY, Zhang R, Chen L, Li BA, Liu SQ, Cao HF, Qiu XH, Shan YF, Yan ZH et al. 2004 Negative regulation of hepatocellular carcinoma cell growth by signal regulatory protein alphal. Hepatology 40 618-628.

Yuan S, Trachtenberg J, Mills GB, Brown TJ, Xu F \& Keating A 1993 Androgen-induced inhibition of cell proliferation in an androgeninsensitive prostate cancer cell line (PC-3) transfected with a human androgen receptor complementary DNA. Cancer Research 53 1304-1311.

Zhu XY, Liu YJ, Lu J \& Xu RB 2004 Knockdown of glucocorticoid receptor expression by RNA interference promotes cell proliferation in murine macrophage RAW264.7 cells. Journal of Steroid Biochemistry and Molecular Biology 92 375-382.

Received in final form 7 August 2008

Accepted 26 August 2008

Made available online as an Accepted Preprint 26 August 2008 